\title{
Research on Future Business Strategy Reform of Gaming Console Brands
}

\author{
Ziyuan Cui ${ }^{1, *}$, a, $\dagger$ Jinghua Jiang ${ }^{2, *}$, b, ${ }^{\dagger}$ Yusen $\mathrm{Zhu}^{3, *, c, \dagger}$ \\ ${ }^{1}$ Frenchay Campus Coldharbour Lane, University of the West of England,BS $161 Q Y$, Bristol, UK \\ ${ }^{2}$ George Mason University, 22030, VA, US \\ ${ }^{3}$ Business School, University of Sussex, BN1 9RH, Brighton, UK \\ ${ }^{*}$ Corresponding author.Email: ${ }^{a} Z i y u a n 2 . C u i @ l i v e . u w e . a c . u k,{ }^{b} j j i a n g 9 @ g m i . e d u,{ }^{c} y z 492 @ s u s s e x . a c . u k$ \\ ${ }^{+}$These authors contributed equally
}

\begin{abstract}
The global games industry has undergone many important and far-reaching changes in the past few decades. In 1979, Atari's first home gaming console came out, and now consoles, personal computers, mobile smartphones or virtual reality, and other gaming platforms have achieved vigorous development in the market. The form of games has changed significantly in different dimensions, such as music, graphics, the complexity of the game types, and the network and sociality. Therefore, grasping the future development trend of the games market is crucial whether for the gaming platform brands' short-term or long-term corporate strategy. Breakthroughs in electronic products and Internet technology have made the content presented by the game more possibilities. Improvements in the performance of personal computers and mobile phones have enabled high-complexity games that could only be run on the consoles can be realized on the two formers. In addition, virtual reality or augmented reality technology has also changed the way players experience games. The popularity of network infrastructure allows players to conduct social activities in the games, rather than just focusing on the game content itself. Therefore, game console brands have various choices to implement their business transformation strategies to meet the challenges brought about by the changing trends of the games industry and the market. They can choose to improve the user experience of console hardware, or develop more social and customized games, or even shift their focus to other gaming platforms.
\end{abstract}

Keywords: Gaming consoles, Business strategy, Hardware improvement, Social experience, Market trends

\section{INTRODUCTION}

\subsection{Background}

The advent of video games has become a new way for people to spend their free time. The game console as the main carrier of game operation has become more efficient with the development of technology. And, due to the changing pace of society brings more opportunities and challenges to the game console industry. The game console should pursue the performance of the game console as before and pay more attention to the portability of the game console or more modern technology. Consoles are still a big market. Almost all of the home gaming consoles brands have to adjust their development strategy to the sharply changing market; thus, they would take advantage of these opportunities and protect themselves from the risks brought by these challenges.

\subsection{Related Research}

Galehantomo and Cunningham have reviewed the development history of video games in their own different researches. Galehantomo summarized the development of various game platforms, including home game consoles, personal computers, and mobile phones. Through a small sample questionnaire survey, this research analyzes the players' views on various attributes of different game platforms. It gets some conclusions, including the dimension of popularity, graphics processing ability, flexibility, price, game richness, satisfaction, and so on. Galehantomo, G. also pointed out that virtual reality / augmented reality technology may become a new generation of the mainstream game platform in the future [1]. While Cunningham made one comprehensive review analysis of the video game industry. The team of Cunningham 
first gives some broad definitions and concepts for video games and host games and discusses their development history. Cunningham then has structurally analyzed the structure of these industries. Secondly, Cunningham uses the Porter's 5 forces model for strategic analysis for major competitors in these industries. Cunningham has particularly emphasized that Online \& Boroadband technology are considered important factors affecting the development of future industries. Cunningham has eventually made some summary work with specific demographics and draws some conclusions, which is that future host game brands need to consolidate and develop their market share by means of key technological upgrades [2]. Furthermore, Nascimento adopted Crandall and Gregory's research results on the console game industry in 2006, reviewed the competition between different brands of seven generations of game consoles in the past, and used microeconomic measurement methods to get some quantitative conclusions about this industry. Nascimento believes that the following views are worth referring to: with the geometric growth of the cost of console games $\mathrm{R} \& \mathrm{D}$, more and more game developers tend to serve the gaming markets of smartphones or tablets, which makes the console gaming market shrinking. Meanwhile, Nascimento pointed out that the income structure of the home gaming industry has also changed. The role played by middlemen is no longer significant. Game's virtual accessories, open-source containers, powerful mobile platforms, and used games retailers are more actively involved in the profit process of this industry, which implies that players have more distribution channels or other platforms to gain access to games [3]. In some more detailed research areas, Kettunen researched how digital sales of video games in the designated console industry could be improved. With a quantitative-analysis questionary survey, the research revealed that there's genuine concern about online safety, hardware restrictions, value creation, and price among console consumers when it comes to buying video games in a digital form. The physical copies were thought to have more value as they could be resold, loaned to friends, or returned to the store for discounts on other games. The physical copies were also thought to have more flexibility when it comes to pricing and discounts. In general, the digital distribution of video games was thought to be more appealing because of it's ease of use, comfortability, and availability of games. Most respondents predicted that digital distribution would be the dominating channel for selling games in the future [4]. This research reveals some negative changes in industry trends because players usually need physical copies to play games on consoles.

In the past, different researchers have conducted specific studies on certain issues, mainly about the consumers' attitude towards the usage experience of consoles. In terms of comfort, Ramolete used quantitative research methods to engage respondents in the physical design assessment of the Nintendo Switch controller configuration. The results of these studies imply that the size and location of the small button are the factors that influence the user experience the most when players participating in controller configuration assessment, which are also reflected in the anthropometric measurements of this study and are also the basis for design suggestions for simulating future predictions. Players responded that inappropriate size and location of small buttons are the most impressive issues to them. Devices like switches may require different anthropometrics to better ergonomic classification, which may be the basis for future research [5]. Regarding usability faults, Ramolete et al. also evaluated the possibility of meeting usability faults of players with different levels of Nintendo Switch usage proficiency and compared among them. The results of the research prove that the initial users of the Nintendo Switch have been observed with all the usability issues and have more failures compared to other users. In contrast, expert users who are familiar with other game consoles and different users have general usability and efficiency improvements. Thus, when designing hardware facilities for gaming consoles, other than models to follow when positioning and designing basic experimental functions and general ergonomic design principles, more important is to create a familiar feeling for initial users by referring to past generations or other similar products [6]. Console performance awareness is another important research issue. MYung et al. made a statistical analysis for situation awareness and presence that affect performance on a handheld game console. Main researching tools are modern handheld devices equipped with high-speed CPUs and 3D graphics accelerators and experience a level similar to virtual desktop reality (VR). The statistical analysis results indicate a positive correlation between the player's intensity of situational awareness during the gaming process and the performance of modern handheld devices. The stronger the processing power of the handheld devices for the game program, the closer the player's experience of the development of the game situation is to the reality so that they will be more immersed in the gaming experience. Research on situational awareness, existence, and performance can even be conducted on research and handheld game consoles. Research is also on handheld game consoles. The research field of handheld awareness and existence can be extended to small displays, such as home gaming consoles [7]. When it turns to market launch, Hendrickson determined ideal launch behaviors for home gaming consoles in the German and Finnish markets. The interactive entertainment industry is growing and will continue to expand. Most of the content in this industry is home game consoles. The theoretical part of this article covers the basic principles of the product and the 
factors to be considered. In addition, there was Porter's launch power and discussed a strategy to participate in the analysis of the interactive entertainment industry. The research data are collected through online questionnaires. Research results supported that factors such as ethnic and social culture, reference groups, perception and attitudes towards products, and motivation influence consumers' buying behavior and the behavior that consumers follow - a summary of the final research results and suggestions for further research [8].

Some scholars have brought about some theoretical framework that would be helpful for console brands to plan for their business strategies. Marchand and Hennig identified a theoretical framework that contains multiple elements concepts by analyzing the internal and external environments of the host game industry. This framework can be used to analyze most of the detailed issues of brand development, as it includes the aspects of the game platform, consumers, recommenders, distribution channels, brand communication channels, etc. Simutaneously, by using this framework, each host gaming brand can confirm which marketing element can provide consumers with the greatest and least replaceable value. Therefore, specific brands can use this framework to confirm the main orientation of their reform strategy. Marchand and Hennig also pointed out that the brands need to find their own challenges and chances to grasp essential creativity or innovation opportunities [9]. Besides, Aleem et al. investigated the effect of business factors on the performance of digital games in the market empirically and to try to find answers to the research questions posed in this study. Empirical investigation results demonstrated that business factors play an important role in digital game performance. The results of the study strongly indicate that customer satisfaction, time to market, monetization strategy, market orientation, and brand name strategy are positively associated with the performance of a digital game organization. The empirical investigation found no strong association or impact between relationship management or innovation and digital game performance. This study is the first of its kind in the field of digital games. It will help and enable organizations to better understand the effectiveness of business factors and their role in terms of game performance in the market. Game development organizations need to consider these various business factors to expand the effects of their current efforts to improve the performance of their developed games in the market [10].

Other studies imply some potential relationship between different console customers and the types of games they like. Manero et al. carried out a small-scale statistical analysis on the relationship between player's characteristics and the type of game they loved. The entire sample includes 754 players. At first, Manero et al. divided the players' group into four sets of different clusters and used the questionnaire to get raw data. Through statistical regression analysis, Manero et al. considered a positive correlation between players' characteristics and game type preferences. This means that if any home gaming console brands want to make a transforming movement for their business structure, they can determine a more specific target consumer group, this can make it more convenient to categorize them and build their own independent players' social communities or provide them with specific types of new games [11]. Apart from that, Nagle et al. designed two studies in order to figure out the relationship between 5 personal traits and customize adaption for shooter games. In Study 1, a linear regression model revealed the relationship between five personality traits with enjoyment and gameplay duration in the four different difficulty levels. In Study 2, this regression model was used to construct a mapping chart that matches different difficulty levels with enjoyment and gameplay duration based on player personality. Dynamic difficulty adjustment was set accordingly based on the mapping chart, which matched difficulty to user performance. Results indicate that enjoyment and gameplay duration was significantly higher in the control group than in the dynamic difficulty adjustment group. The present work highlights the importance of difficulty adaptation as a game mechanic and suggests that personality could also be related to other game mechanics. Accordingly, as a result, a framework for personality-based game mechanics customization is proposed to foster future research. This research might be helpful for home gaming console brands to develop their next generation of products in terms of their certain targeted audience, which means that they can adapt new games for the consumers with different personality traits [12].

\subsection{Objectives}

At three different levels, namely the analysis of the development trend of the game market, the improvement of the console's hardware capabilities, and the transformation of the business model, the game console brand can arrange specific future strategies for itself. These strategies may involve overall strategic transmission based on market and industry trends, consideration of game console hardware and performance improvement directions, or changes in game types and profit methods. This exploratory research aims to discuss the potential choices of traditional game console brands such as Sony, Xbox, and Nintendo for these three levels. 


\section{MARKET AND TRENDS ANALYSIS}

\subsection{Development trend of home game consoles}

Home game consoles have gone from the graphically humble Atari 2600 to today's graphically polished Playstation 5. The console industry uses better technology to make games more realistic, with better effects and better stories. With the development of The Times, consumers are no longer satisfied with the sensory stimulation brought by the initial movement trajectory of a few small pixel blocks. Instead, they pursue to shuttle in the visual impact brought by more exquisite game images and special effects. Historical console industry trends have focused on better graphics and some innovative console features. With the development of the Internet and the rapid pace of society, players no longer have time to spend on their home consoles and are more concerned with spending fragmented time and interacting with other players. The console industry has changed dramatically.

\subsection{The impact of games genre changes on game consoles}

In the past, game design was limited to single-player games because of the crude hardware and network equipment. So that, game makers focus on story development and gameplay innovation. Previous games didn't have any player-to-player interaction or antagonism. As a result, the overall number of gamers is growing slowly. Consoles have become more powerful as technology has grown, so games have become more realistic and expressive. Cinematic, immersive gaming experiences are beginning to be promoted by game makers. During this period, a large number of gamers grew.

With the development of the Internet, information was transferred from one console to another faster, so the next stage of gaming was more interactive and competitive between players. In the following period, the rapid growth of online games quickly grabs the market share from console games. Today, traditional games are going down three paths. The first is to continue making big TRIPLE-A games, and the second is to start developing new technologies for VR-type games. The third is to adapt to the consumer hobby into the development of mobile games. The third method seems to be the most profitable in terms of revenue power.

Table 1. Games and interactive media earnings rose $12 \%$ to $\$ 139.9 \mathrm{~B}$ in 2020

\begin{tabular}{cc}
\hline Digital Games & Market Value \\
\hline Mobile & $\$ 73.8 \mathrm{~B}$ \\
PC & $\$ 33.1 \mathrm{~B}$ \\
Console & $\$ 19.7 \mathrm{~B}$ \\
\hline
\end{tabular}

\subsection{Future console market forecast}

When marketers combine historical console and gaming trends into analysis, they will find the truth that consoles will either upgrade their hardware to make the graphics and effects of the games cooler, or they will use new technology to make the games appear in a new way. Games went from single-player games with better graphics and special effects to player-to-player interactions and confrontations. So in combination with the previous analysis of historical console trends, there are two ways that games can develop to win the market. The first is to develop VR devices and take gaming to the next level through technological innovation, which is truly immersive. Despite its current meager array of devices and games, VR is undeniably going to be the next big thing in the gaming industry. The second is the mobile gaming market, which caters to the needs of players and their increasingly fragmented time. Most of the top-grossing games in the market are mobile and competitive games, proving that the mobile market and esports have great potential.

Table 2. TOP 10 Free to play Games

\begin{tabular}{|c|c|c|c|c|}
\hline Rank & Title & Publisher & Genre & Revenue \\
\hline 1 & Honor of kings & Tencent & MOBA & $\$ 2.45 \mathrm{~B}$ \\
\hline 2 & Peacekeeper Elite & Tencent & Shooter & $\$ 2.32 \mathrm{~B}$ \\
\hline 3 & Roblox & Roblox Corporation & Simulation & $\$ 2.29 \mathrm{~B}$ \\
\hline 4 & Free Fire & Garena & Shooter & $\$ 2.13 \mathrm{~B}$ \\
\hline 5 & Pokemon Go & Niantic.Inc. & Action-Adventure & $\$ 1.92 \mathrm{~B}$ \\
\hline 6 & League of Legends & Riot. Games, Tencent & MOBA & $\$ 1.75 B$ \\
\hline 7 & Candy Crush Sage & King, Activision blizzard & Puzzle & $\$ 1.66 \mathrm{~B}$ \\
\hline 8 & AFK Arena & Lilith Games & Strategy & $\$ 1.45 B$ \\
\hline 9 & GardenScapes - New Acres & Playrix Games & Puzzle & $\$ 1.43 \mathrm{~B}$ \\
\hline 10 & Dungeon Fighter Online & Nexon & RPG & $\$ 1.41 \mathrm{~B}$ \\
\hline
\end{tabular}




\section{PRODUCT IMPROVEMENT STRATEGY ANALYSIS}

\subsection{Controller Comfort and Performance Awareness}

The design and performance of game console controllers are critical to the player's gaming experience. In order to maximize the player's positive experience and perception of controllers, it is necessary to use ergonomic knowledge to design the controller's appearance and component configuration. Factors such as the length of the grips, the size and location of the buttons, the sensitivity of components, and creative or innovative conceptual appearance all impact the player's attitude towards the controllers. The performance of the controller and the console could directly affect the player's immersive experience of games because the better the performance can make the player have a better perception of the gaming situation, which means that players can more clearly know what is going on in the games. Virtual reality/augmented reality is an emerging technology that can be used as an auxiliary means to enhance the player's state perception to a certain extent. The market for virtual reality /augmented reality technology is also growing in recent years.

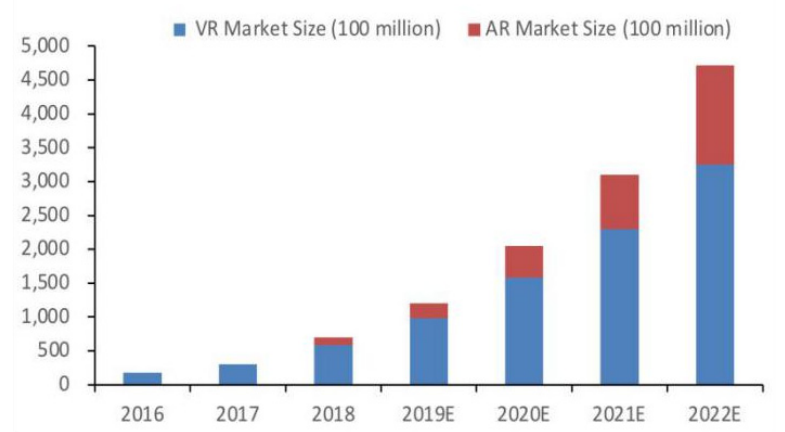

Figure 1. Global VR/AR industry market size and forecast

\subsection{Consumer Behaviour and Hardware}

Research on consumer behavior is also beneficial to product improvement. Consumer behavior can be viewed as a complex system affected by many different factors, some of which are at a macro level, such as social culture. In contrast, other factors are related to individual ones, such as reference group, family, and income level. The hardware facilities of each generation of game consoles have improved to a larger or smaller extent, but most of these improvements are based on the consideration of improving the gaming experience or reducing costs. Some innovative viewpoints believe that the arrangement of gaming console hardware can also partially conform to consumer behavior issues. For instance, based on the cultural background and income level of target consumers in certain market segmentation, the hardware appearance design could be determined on those which are more in line with those consumers' aesthetics.

\subsection{Usability Analysis and Strategy}

The impact of the usability of gaming console controllers on the player's gaming experience cannot be ignored in particular. This issue involves whether the player can master the usage of controllers as quickly as possible. Therefore, the use complexity, functional complexity, compatibility, and faults probability of controllers are all important factors that need to be considered when developing the controller techniques. For initial players to adapt to operation pattern of controllers in the shortest time, the most reliable way is to continue the settings of previous generations of products or refer to other similar products, rather than to carry out excessive innovation. There is evidence that the higher the controller usability, the better the business performance of the gaming consoles. A typical example is that Nintendo's two similar products, Nintendo Switch and Nintendo 3DS. While Nintendo Switch's controller is easier to operate than the $3 \mathrm{DS}$, so its sales are also higher.

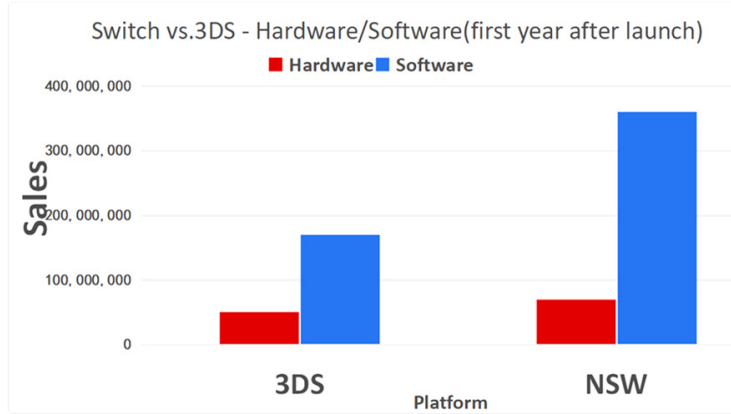

Figure 2. Nintendo switch and Nintendo switch 3DS sales comparison

\section{BUSINESS PATTERN}

\subsection{Transformation of business pattern}

The business transformation of console game brands may need to involve many different factors into consideration. This may include the selection of new platforms. For example, companies could consider transferring their core game business to mobile phones or personal computer platforms. It may also be engaged with the transformation of the core content of the games. As network infrastructure becomes more and more common, companies can try to make more online games that are more based on social experiences rather than stand-alone games that focus too much on the content itself. And most importantly, whether the companies need to create or emulate some unusual profit models is the issue that they should treat as a certain critical 
transformation process. For instance, the profit of traditional stand-alone games mainly comes from the one-time games buyout. Players can own the game once they pay, while the more popular profit model adopted for social games now is mainly completely different The latter allows players to get the game for free, but they can pay for some paid items to enhance their personal or social experience.

Table 3. Comparison between two types of games

\begin{tabular}{ccc}
\hline Item & Traditional single games & Online social games \\
\hline Main sources from gaming experience & $\begin{array}{c}\text { Games content such as plot, } \\
\text { gameplay, music, picture quality, } \\
\text { game journey }\end{array}$ & Social interaction with other players \\
& \multicolumn{2}{c}{$\begin{array}{c}\text { gamen } \\
\end{array}$}
\end{tabular}

Gaming platforms

Profiting pattern
Mainly home consoles

One Buy-out piece
Personal computers, mobile smart phones

Free games but optional items inside that players can choose to purchase to enhance their gaming experience

\subsection{Innovation and customer satisfaction}

Business transformation requires companies to take innovative measures. But companies must realize that innovation is a means rather than an end. Regardless of the specific reform plan adopted, console game brands should always focus on customers and improve customer experience and satisfaction as the goals of the reform. Innovation does not necessarily bring about commercial success because the key to success lies in whether the product meets customers' expectations. Historically, there have been many failed innovation cases in the game industry, whether it is about the games or the platform innovation. Examples of the former include Call of Duty 15, which has been criticized for deleting the single-player campaign plot that was the core selling point of the game series in the past all the time. The latter example includes the commercial failure of PS3 due to the high R\&D costs caused by the immaturity of many technologies. Innovation should be used to make up for the shortcomings of existing products or business models, not for risky commercial experiments.

\subsection{Games design of certain orientation for different players}

In fact, the personality traits or characteristics of different players may be related to the type of games they like. This means that console game brands need to consider their target consumers' groups when designing new game products. For example, if a console game brand wants to develop mobile games or social games, they need to describe a new group of players that are different from single-player gamers. This new group may not be addicted to the games. They are just used to taking fragmented time to conduct social activities in the game or obtain temporary pleasure. Therefore, console game brands need to clarify their product needs and provide consistent services and marketing activities. Even console game brands can further divide the target group and design more personalized products for smaller clusters.

\section{CONCLUSION}

This article carries out some analysis on the future business strategy development of game console brands. According to previous research and business reports, the traditional console game industry market is currently shrinking, and game developers are increasingly inclined to cooperate with personal computers or mobile phone platforms. The research indicates that console game brands can choose a variety of strategies at different levels to cope with the challenges brought about by changes in the market and industry trends, such as shifting the industry's focus to other game platforms, improving the console's hardware facilities, or adding more social and customized games. Future research can be aimed to explore the prospects and feasibility of various strategies in more detail and bring valuable views through more market data and technical discussions.

\section{REFERENCES}

[1] Nascimento, A.,M. (2013). A microeconomic analysis of the competition in the home console videogame industry. Retrieved from: http://hdl.handle.net/1721.1/81005

[2] Kettunen, J. (2017). Console gaming : an analysis of how digital sales can be increased from both an industry and consumer perspective. Retrieved from: http://urn.fi/URN:NBN:fi:amk-201704275609

[3] Galehantomo, G. (2015). Platform Comparison Between Games Console, Mobile Games And PC Games. Sisforma, 2015-2(1), 23-26. 
[4] Cunningham, A. et al. (2008). Video games industry overview: an analysis of the current market and future growth trends. Retrieved from: holgerlanglotz.de/downloads/BU4510_VideoGame sIndustry_LanglotzEtAl.pdf

[5] Ramolete, G., L. et al.(2020). Physical design assessment of the Nintendo Switch controller configurations. Retrieved from: https://www.researchgate.net/publication/3425971 20_Physical_Design_Assessment_of_the_Nintend o_Switch_Controller_Configurations

[6] Ramolete, G., L. et al.(2020). Usability and gaming experience assessment of the Nintendo Switch user interface by Filipino users. Retrieved from: https://www.researchgate.net/publication/3425971 20_Physical_Design_Assessment_of_the_Nintend o_Switch_Controller_Configurations

[7] MYung, R. et al. (2008). A study of relationships between situation awareness and presence that affect performance on a handheld game console. Retrieved from: https://www.researchgate.net/publication/2641365 05_A_Study_of_Relationships_among_Situation_ Awareness_Presence_and_Performance_on_a_Han dheld_Game_Console

[8] Hendrickson, E.(2018). Comparison of product launch strategies for home gaming consoles. Retrieved from: http://urn.fi/URN:NBN:fi:amk201803273859

[9] Marchand, A. \& Hennig-Thurau, T. (2013). Value creation in the video game industry: industry economics, consumer benefits, and research opportunities. Journal of Interactive Marketing, 27(2013), 141-157.

[10] Manero, B. et al. (2016). An instrument to build a gamer clustering framework according to gaming preferences and habits. Computers in Human Behavior, 62(2016), 353-363.

[11] Aleem, S., Capretz, L., F. \& Ahmed, F. (2016). Empirical investigation of key business factors for digital game performance. Entertaiment Computing, 13(2016), 25-36.

[12] Nagle, A., Wolf, P. \& Riener, R. (2016). Towards a system of customized video game mechanics based on player personality: relating the big five personality traits with difficulty adaptation in a first-person shooter game. Entertainment Computing, 13(2016), 10-24. 